${ }^{1}$ Internal Medicine Department, Faculty of Medicine, Alexandria University, Alexandria, Egypt

IInternal Medicine Department, Medical Research Institute, Alexandria University, Alexandria, Egypt

${ }^{3}$ Chemical Pathology Department, Medical Research Institute, Alexandria University, Alexandria, Egypt

\title{
Serum Long Non-Coding RNAs in Diabetic Kidney Disease
}

\section{ABSTRACT}

Background: Diabetic kidney disease (DKD) afflicts almost $40 \%$ of type 2 diabetes mellitus (T2DM) patients and may progress to end-stage renal disease despite multifactorial management. Urinary albumin/creatinine ratio (UACR) and estimated glomerular filtration rate (eGFR) are not sufficiently sensitive or specific for early diagnosis or recognition of patients at higher risk of cardiorenal complications. Long non-coding RNAs (IncRNAs) emerged as key regulators of major DKD signaling pathways. Their potential role as novel biomarkers or therapeutic targets in DKD remains to be established. Methods: We studied 4 groups of 25 adults each. G1, 2, 3 had T2DM and normal, moderately-increased, and severely-increased UACR, respectively. G4 were healthy controls. Participants underwent clinical assessment and analysis of blood picture, fasting serum glucose, glycated hemoglobin, lipid profile, hepatic transaminases, albumin, urea, and creatinine. By real-time quantitative polymerase chain reaction, we assessed relative serum expression levels of the IncRNAs plasmacytoma variant translocation-1 gene (PVT1), metastasis-associated lung adenocarcinoma transcript-1 (MALAT1), and cancer susceptibility candid-2 gene (CASC2).

Address for correspondence:

Dr. Yaser Aly Ammar, Assistant Professor of Internal Medicine

Medical Research Institute, Alexandria University

Alexandria, Egypt

e-mail: yaser_ammar88@yahoo.com

Clinical Diabetology 2022, 11; 2: 80-89

DOI: $10.5603 /$ DK.a2022.0007

Received: 26.08 .2021

Accepted: 22.12.2021
Results: Serum PVT1 was significantly higher in diabetic patients compared with controls, and significantly higher in $\mathrm{G} 2$ compared with the other patients' groups. Serum MALAT1 and CASC2 showed no statistically significant association with the occurrence of T2DM, DKD, or other major diabetic complications. Increased serum creatinine was the only significant predictor of increased UACR.

Conclusions: Increased serum PVT1 is a reliable early biomarker of DKD progression. It may provide a plausible therapeutic approach. The involvement of MALAT1 and CASC2 in DKD pathogenesis is less established. Further large-scale prospective studies are warranted. (Clin Diabetol 2022, 11; 2: 80-89)

Keywords: albuminuria, diabetes mellitus, diabetic kidney disease, long non-coding RNA, polymerase chain reaction

\section{Introduction}

Diabetes mellitus (DM) comprises a group of hyperglycemic metabolic disorders resulting from defective insulin secretion and/or action [1]. It is a rapidly growing global health problem, with dreadful complications on almost all body systems, and major socio-economic impact, particularly in low and middle-income countries [2]. More than $90 \%$ of cases are classified as type 2 (T2DM), which has been particularly surging in Egypt, in parallel with the prevalent obesity, low physical activity, and suboptimal management [3]. Up to $40 \%$ of diabetics develop diabetic kidney disease (DKD), a major cause of the end-stage renal disease (ESRD) worldwide, 
associated with significantly increased cardiovascular morbimortality. [1]. A broadening array of established and novel risk markers may be utilized for DKD diagnosis and staging; but clinical practice relies mainly on measurement of albuminuria, mainly urinary albumin/ creatinine ratio (UACR), and estimated glomerular filtration rate (eGFR) [4]. However, the classical model of DKD starting with glomerular hyperfiltration and increased UACR, followed by declining eGFR has been greatly challenged; and many other different trajectories of albuminuria and renal function alteration were described in biopsy-proven DKD patients [5]. Moreover, DKD categorization by UACR is hampered by generally low sensitivity/specificity and significant intraindividual variability [6]; whereas creatinine-based eGFR formulas are perplexed by methodological limitations, nonrenal confounders, and inaccuracies at the near-normal range [4]. Standard of care management including control of hyperglycemia, hypertension, dyslipidemia, and all known modifiable factors might still fail to prevent chronic kidney disease (CKD) progression $[1,4]$. In the modern era of precision (individualized) medicine, it is highly required to identify novel diagnostic markers, preferably with therapeutic potential.

Genetic/epigenetic factors significantly impact DKD occurrence and progression, as dictated by its familial clustering and unequal prevalence among different ethnic groups. Only $2 \%$ of human genomic DNA is proteincoding; the major remainder is transcribed in a highly cell/tissue-specific fashion into non-coding RNA. Initially thought of as junk or functionless segments, noncoding RNA was found to constitute a major network of epigenetic control of various biological processes in health and disease [7]. The main classes of functional noncoding RNAs are micro RNA (20-22 nucleotides length), circular RNA (circRNA), and long noncoding RNA (> 200 nucleotides length, IncRNA) [8]. IncRNAs were detected in the nucleus, cytoplasm, and various tissue fluids. A plethora of recent studies described the critical roles of IncRNA in regulating beta-cell function, insulin resistance, and the alterations of renal cell populations induced by high glucose exposure (HGE) and inflammation [9]. They may behave like sponges, repressors, or activators of miRNAs and inflammatory or fibrogenic cytokines involved in the major pathways of DKD progression [10]. Some IncRNAs are induced by HGE and contribute to podocyte and tubular damage through well-characterized signaling pathways [9]. These include the plasmacytoma variant translocation 1 (PVT1) transcript, which was the first IncRNA reported in association with DKD progression [11], and the metastasis-associated lung adenocarcinoma transcript 1 (MALAT1), a widely distributed IncRNA that promotes renal tubular epithelial pyroptosis [12]. On the other hand, the cancer susceptibility candid 2 (CASC2) gene is downregulated by HGE and may indeed ameliorate HGE- induced renal injury and improve DKD [13].

Therefore, IncRNAs have recently emerged as novel markers for early diagnosis and prognosis of DKD; and might also provide potential therapeutic targets [9]. However, a recent systematic review found that the impact of most IncRNAs studied in DM was inconsistent, due to the absence of standardized nomenclature and quantification techniques, as well as a paucity of studies evaluating a given IncRNA in specific body tissue or fluid [14]. Moreover, most IncRNA studies in the context of DKD were performed on in-vitro models or experimental animals, with relatively few human studies [9]. Since IncRNAs are relatively stable and readily detectable by realtime quantitative polymerase chain reaction (RT-qPCR) in serum samples [15], we assumed that further IncRNAs serum studies in T2DM patients might approximate the gap between IncRNA research and practice.

\section{Methods}

This was a cross-sectional, case-control study, conducted in accordance with the provisions of the Declaration of Helsinki (revised in 2008) and approved by the Ethics Committee of the Faculty of Medicine, Alexandria University, Egypt. Adult subjects accepting to sign a written informed consent were recruited from the outpatient clinics of Alexandria Main University Hospital and Medical Research Institute. Type 2 DM was diagnosed according to the 2021 American Diabetes Association criteria [1]. Urinary albumin/creatinine ratio was assessed in a morning urine sample and, if increased, confirmed in 2/3 samples over 3-6 months [16]. Participants were accordingly classified into 4 study groups of 25 adults each:

- Group 1 (G1): T2DM patients with normal UACR (< $30 \mathrm{mg} / \mathrm{gm}$ )

- Group 2 (G2): T2DM patients with moderatelyincreased UACR ( $\geq 30$ and $<300 \mathrm{mg} / \mathrm{gm}$ )

- Group 3 (G3): T2DM patients with severely-increased UACR ( $\geq 300 \mathrm{mg} / \mathrm{gm}$ )

- Group 4 (G4): healthy controls matching with the diabetic patients

We excluded patients with acute kidney injury, renal stones, urinary tract infection, recent nephrotoxic drug exposure, CKD of non-diabetic etiology, or GFR $\leq$ $30 \mathrm{ml} / \mathrm{min} / 1.73 \mathrm{~m}^{2}$. All subjects underwent a detailed clinical assessment including a history of diabetes and its complications, measurement of arterial blood pressure, body mass index, waist circumference, cardiovascular and neurological assessment, and fundus examination. 
Table 1. Real-Time Quantitative Polymerase Chain Reaction

\begin{tabular}{|c|c|c|c|c|}
\hline \multicolumn{5}{|l|}{ A. Primers used } \\
\hline Gene & Forward primer & \multicolumn{3}{|c|}{ Backward primer } \\
\hline GAPDH & 5'-GAAGGTGAAGGTCGGAGTCAAC-3' & \multicolumn{3}{|c|}{ 5'-CAGAGTTAAAAGCAGCCCTGGT -3' } \\
\hline PVT1 & 5'-TGAGAACTGTCCTTACGTGACC - $3^{\prime}$ & \multicolumn{3}{|c|}{ 5' -AGAGCACCAAGACTGGCTCT -3' } \\
\hline MALAT1 & 5'-AAAGTCCGCCATTTTTGCCAC-3' & \multicolumn{3}{|c|}{ 5'-ACAACTCGCATCACCGGAAT-3' } \\
\hline CASC2 & 5'-GCACATTGGACGGTGTTTCC- $3^{\prime}$ & \multicolumn{3}{|c|}{ 5'-CCCAGTCCTTCACAGGTCAC-3' } \\
\hline \multicolumn{5}{|c|}{ B. Thermal cycling conditions } \\
\hline Step & Temperature & & Time & Number of cycles \\
\hline Initial denaturation & $95^{\circ} \mathrm{C}$ & & $10 \mathrm{~min}$ & 1 \\
\hline Denaturation & $95^{\circ} \mathrm{C}$ & & $15 \mathrm{~s}$ & 40 \\
\hline \multirow[t]{4}{*}{ Annealing } & GAPDH & $64^{\circ} \mathrm{C}$ & $30 \mathrm{~s}$ & \\
\hline & MALAT1 & $62^{\circ} \mathrm{C}$ & $30 \mathrm{~s}$ & \\
\hline & PVT1 & $56^{\circ} \mathrm{C}$ & $30 \mathrm{~s}$ & \\
\hline & CASC2 & $58^{\circ} \mathrm{C}$ & $30 \mathrm{~s}$ & \\
\hline Extension & & $72^{\circ} \mathrm{C}$ & $30 \mathrm{~s}$ & \\
\hline
\end{tabular}

CASC2 - cancer susceptibility candidate 2; GAPDH — glyceraldehyde-3-phosphate dehydrogenase; MALAT1 — metastasis-associated lung adenocarcinoma transcript 1; PVT1 - plasmacytoma variant translocation 1 gene

After an overnight fast of 10-12 hours, 10 milliliters of venous blood were withdrawn from each subject and divided into:

- EDTA vacutainer tube (1) for measurement of complete blood count and glycated hemoglobin (HbA1c) using XN-350 (Sysmex);

- Plain vacutainer tube. Blood was allowed to clot for 30 minutes at room temperature, then centrifuged at $4000 \mathrm{rpm}$ for 15 minutes. Separated serum was used for the determination of fasting glucose, total cholesterol (TC), triglycerides (TGs), low-density lipoproteins (LDLs), high-density lipoproteins (HDLs), alanine and aspartic transferases (ALT, AST), albumin, urea, and creatinine [17]. eGFR was calculated by the CKD-epidemiology collaboration (CKD-EPI) equation [18]. Chemical analysis was done using Olympus AU400 clinical chemistry analyzer (Beckman Coulter Inc.);

- EDTA vacutainer tube (2) for IncRNA gene expression studies.

\section{IncRNA gene expression studies}

1) RNA extraction:

Total serum RNA was isolated using miRNeasy Mini Kit (QIAGEN) Cat No./ID: 217004, according to the manufacturer's protocol. Briefly, $5 \mu$ l of $5 \mathrm{nM}$ Syncel-miR-39 (miScriptmiRNA Mimic) was added to each sample as a spike-in control, and then total RNA was purified from $400 \mu \mathrm{l}$ of a sample. The upper aqueous phase containing RNA was extracted, and ethanol was added to provide appropriate binding conditions. The sample was then applied to the RNeasy Mini spin column, where the total RNA binds to the membrane and contaminants are washed away.

The concentration of total RNA in the extract was determined by Thermo Scientific NanoDrop ${ }^{\mathrm{TM}} 1000$ Spectrophotometer (Thermo Scientific, USA), where the absorbance at $260 \mathrm{~nm}$ (A260), at 280 (A280), and at 230 (A230) were measured. The ratios of (A260/A280) and (A260/A230) were used as measures of nucleic acid purity [19].

The elution tubes were capped and stored at $-80^{\circ} \mathrm{C}$.

2) Reverse transcription (RT) (complementary DNA [cDNA] synthesis):

Complementary DNA synthesis was done using High-Capacity cDNA RT Kit (Thermo Scientific, USA, \# 4368814), according to manufacturer's protocol. Total volume was $20 \mu \mathrm{l}$ and RT reaction was carried out on Primus 25 advanced $\hat{A} \circledast$ Thermocycler, (Pedqlab, Switzerland). Samples were incubated for $10 \mathrm{~min}$ at $25^{\circ} \mathrm{C}, 120 \mathrm{~min}$ at $37^{\circ} \mathrm{C}$ then terminated by heating at $85^{\circ} \mathrm{C}$ for $5 \mathrm{~min}$. CDNA was stored at $-20^{\circ} \mathrm{C}$ till it was used for $q P C R$ reaction.

3) RT-qPCR:

The relative quantitation of PVT1, MALAT1 and CASC2 IncRNA transcripts was performed by qPCR (Eco illumina, USA) using Maxima SYBR Green qPCR master mix (Thermo Scientific, USA, \# K0251) using specific primer sequences and following specific thermal cyclic conditions (Tab. 1).

4) Quantitation:

Relative Quantitation of PVT1, MALAT1, and CASC2 was calculated using the comparative $\mathrm{Cq}$ method (2- $\Delta \Delta \mathrm{Cq})$ after normalization for the expression of 
$\beta$ - Actin (glyceraldehyde-3-phosphate dehydrogenase [GAPDH]) as an endogenous reference gene [20]. Relative gene expression in relation to GAPDH was log transformed so that values above or below zero are indicative of relative over- or under-expression (respectively) of the target gene.

\section{Statistical methods}

Data were analyzed using SPSS software package version 20 (SPSS Inc., Chicago, Illinois, USA). Categorical data were expressed as absolute numbers (percentages) and compared by Chi-square or Fisher exact test. Continuous data were tested for normality using the Shapiro-Wilk test. Parametric data were presented as mean \pm SD and compared by analysis of variance (ANOVA) or independent t-test. Non-parametric data were presented as median (interquartile range) and compared by Kruskal-Wallis test. Post-hoc analysis was performed for pairwise comparisons. Odds ratios and 95\% confidence intervals were calculated for the association of over- or under-expressed IncRNA serum levels and the occurrence of T2DM and its complications. Pairwise correlations were tested by Spearman's rank correlation coefficient. Multivariate linear regression analysis was employed to identify the most significant predictors of increased UACR. Significance was judged at the $5 \%$ level.

\section{Results}

Controls were matching with the 3 patients' groups regarding sex and prevalence of smoking, but they had significantly younger age, and lower blood pressure (systolic, diastolic, and mean) (Tab. 2). Patients with increased $\operatorname{UACR}(G 2,3)$ had significantly more frequent diabetic complications (coronary artery disease [CAD], peripheral arterial disease [PAD], diabetic retinopathy, diabetic neuropathy), hypertension, and longer duration of diabetes, compared with normoalbuminuric patients (G1). G3 patients had significantly higher body mass index (BMI) compared with G1. There were no significant differences among the 4 study groups regarding platelet count, lipid profile, hepatic transaminases, and log serum IncRNAs (as 4 separate groups) (Tab. 3). However, pairwise comparisons of serum PVT1 revealed that controls had a significantly lower mean level compared with T2DM patients (G1 2, 3 collectively) (Fig. 1); and $\mathrm{G} 2$ patients had a significantly higher mean level compared with other T2DM patients (G1, 3 collectively). Controls had significantly higher hemoglobin, and lower $\mathrm{HbA1c}$ and fasting serum glucose (FSG) compared with the patients ' groups. G3 patients had significantly higher serum creatinine and UACR and significantly lower eGFR compared with the other groups. We found no statistically significant association between deviant serum IncRNA levels and the occurrence of T2DM (Tab. 4) or its complications (Tab. 5).

Within the diabetic patients, the studied IncRNAs showed no significant correlations with other study parameters (Tab. 6). In multivariate analysis inclusive of diabetic patients only, increased serum creatinine was the only significant predictor of increased UACR (Tab. 7).

\section{Discussion}

The prevalence of ESRD attributed to T2DM has been increasing in recent years due to increased prevalence and decreasing age at onset of T2DM. Renal biopsy may be required to precisely diagnose and grade DKD, but it has a significant risk of complications and might not be readily acceptable by patients and attending physicians [1]. Search for novel DKD markers with diagnostic/therapeutic potential has focused on IncRNAs as they have high tissue specificity and are potentially readily detectable in serum and body fluids $[9,10]$. In the present study, we assessed the serum levels of 3 key IncRNAs in healthy subjects and 3 groups of T2DM patients defined by the typical UACR strata adopted by most other studies. Relative gene expression data were log-transformed to remove bias originating from inequivalence of the linear scale extent around the equivalence point, which would have made the data markedly positively skewed and not normally distributed [21].

The main finding of the present study concerns the InCRNA PVT1, which, compared with the housekeeping GAPDH gene, was underexpressed in controls and over-expressed in all T2DM patients' groups, with a statistically significant difference (Fig. 1). Moreover, G2 patients (with early DKD) had significantly higher serum PVT1 compared with other patients (having either no or advanced DKD). Tens of thousands of IncRNAs are expressed in the kidney; and an expanding array of them was reported in association with T2DM and DKD [9, 10]; but PVT1 was the first IncRNA to be described in relation to DKD progression in two canonical human single nucleotide polymorphisms (SNPs) gene association studies; which covered the two major types of diabetes, and were conducted in two ethnically divergent populations (Pima Indians and Caucasians), supporting a true association of DKD progression to ESRD with PVT1 variants rather than with another putative nearby gene locus [11, 22]. Two recent studies reported that PVT1 is highly expressed in sera of DKD patients and human renal mesangial cells under conditions of HGE. Knockdown of PVT1 in these studies alleviated HGE-induced mesangial proliferation and fibrosis $[23,24]$. In a recently inaugurated com- 
Table 2. Demographics and Clinical Data of the Four Study Groups

\begin{tabular}{|c|c|c|c|c|c|c|c|}
\hline Parameter & $\begin{array}{c}\text { G1 (normal } \\
\text { UACR) }\end{array}$ & $\begin{array}{l}\text { G2 (moderately } \\
\text { increased UACR) }\end{array}$ & $\begin{array}{c}\text { G3 (severely } \\
\text { increased UACR) }\end{array}$ & $\begin{array}{c}\text { G4 } \\
\text { (controls) }\end{array}$ & $\begin{array}{c}\text { Statistical } \\
\text { test }\end{array}$ & $\mathbf{P}$ & $\begin{array}{c}\text { Pairwise } \\
\text { comparisons }\end{array}$ \\
\hline \multicolumn{8}{|l|}{ Sex } \\
\hline Males & $9(36 \%)$ & $10(40 \%)$ & $13(52 \%)$ & $13(52 \%)$ & $\chi^{2}$ & 0.699 & \\
\hline Females & $16(64 \%)$ & $15(60 \%)$ & $12(48 \%)$ & $12(48 \%)$ & & & \\
\hline Age [years] & $53.4 \pm 8.9$ & $60 \pm 12$ & $56 \pm 10.8$ & $43 \pm 10$ & ANOVA & $<0.001$ & G4 < others \\
\hline BMI $\left[\mathrm{kg} / \mathrm{m}^{2}\right]$ & $\begin{array}{c}28 \\
(26.1-30.8)\end{array}$ & $\begin{array}{c}30 \\
(27.7-35.9)\end{array}$ & $\begin{array}{c}32 \\
(29.1-38.1)\end{array}$ & $\begin{array}{c}28 \\
(26.4-29)\end{array}$ & KWT & $0.003^{* *}$ & $\mathrm{G} 3>\mathrm{G} 1 ; \mathrm{G} 4$ \\
\hline $\begin{array}{l}\text { WC }[\mathrm{cm}] \\
\text { (males) }\end{array}$ & $\begin{array}{c}86 \\
(84-94)\end{array}$ & $\begin{array}{c}89 \\
(83.5-90)\end{array}$ & $\begin{array}{c}88 \\
(75-96)\end{array}$ & $\begin{array}{c}80 \\
(75-82)\end{array}$ & $\mathrm{KWT}$ & $0.014^{*}$ & $\mathrm{G} 2>\mathrm{G} 4$ \\
\hline $\begin{array}{l}\text { WC }[\mathrm{cm}] \\
\text { (females) }\end{array}$ & $\begin{array}{c}82 \\
(79.3-87.8)\end{array}$ & $\begin{array}{c}86 \\
(79-89)\end{array}$ & $\begin{array}{c}91 \\
(84-92.8)\end{array}$ & $\begin{array}{c}80 \\
(74-83.3)\end{array}$ & KWT & $0.042 *$ & $\mathrm{G} 3>\mathrm{G} 4$ \\
\hline DOD [years] & $7(6-10)$ & $11(8-15)$ & $10(7-19)$ & & $\mathrm{KWT}$ & $0.039 *$ & $\mathrm{G} 1<\mathrm{G} 2 ; \mathrm{G} 3$ \\
\hline \multicolumn{8}{|l|}{ Smoking } \\
\hline Yes & $6(24 \%)$ & $5(20 \%)$ & $8(32 \%)$ & $5(20 \%)$ & $\chi^{2}$ & 0.725 & \\
\hline No & $19(76 \%)$ & $20(80 \%)$ & $17(68 \%)$ & $20(80 \%)$ & & & \\
\hline \multicolumn{8}{|l|}{ Hypertension } \\
\hline Yes & $5(20 \%)$ & $9(36 \%)$ & $13(52 \%)$ & $1(4 \%)$ & Fisher's exact test & $0.001 * *$ & $\mathrm{G} 2 ; 3>\mathrm{G} 1 ; 4$ \\
\hline No & $20(80 \%)$ & $16(64 \%)$ & $12(48 \%)$ & $24(96 \%)$ & & & \\
\hline $\mathrm{SBP}[\mathrm{mmHg}]$ & $\begin{array}{c}125 \\
(120-135)\end{array}$ & $\begin{array}{c}140 \\
(130-150)\end{array}$ & $\begin{array}{c}150 \\
(140-160)\end{array}$ & $\begin{array}{c}115 \\
(110-120)\end{array}$ & KWT & $<0.001^{* *}$ & $\begin{array}{c}\text { G4 < others } \\
\text { G3 > G1 }\end{array}$ \\
\hline $\mathrm{DBP}[\mathrm{mmHg}]$ & $80(80-90)$ & $85(80-90)$ & $90(85-90)$ & $70(70-80)$ & KWT & $<0.001^{* *}$ & G4 < others \\
\hline $\mathrm{MBP}[\mathrm{mmHg}]$ & $\begin{array}{c}95 \\
(93.3-103.3)\end{array}$ & $\begin{array}{c}103.3 \\
(93.3-106.7)\end{array}$ & $\begin{array}{c}106.7 \\
(103.3-113.3)\end{array}$ & $\begin{array}{c}83.3 \\
(83.3-91.7)\end{array}$ & $\mathrm{KWT}$ & $<0.001 * *$ & $\begin{array}{c}\text { G4 < others } \\
\text { G3 > G1 }\end{array}$ \\
\hline \multicolumn{8}{|l|}{ CAD } \\
\hline Yes & $1(4 \%)$ & $8(32 \%)$ & $6(24 \%)$ & $0(0 \%)$ & Fisher's exact test & $0.002 * *$ & $\mathrm{G} 2 ; 3>\mathrm{G} 1 ; 4$ \\
\hline No & $24(96 \%)$ & $17(68 \%)$ & $19(76 \%)$ & $25(100 \%)$ & & & \\
\hline \multicolumn{8}{|l|}{ PAD } \\
\hline Yes & $1(40 \%)$ & $3(12 \%)$ & $6(24 \%)$ & $0(0 \%)$ & Fisher's exact test & $0.025^{*}$ & $\mathrm{G} 2 ; 3>\mathrm{G} 1 ; 4$ \\
\hline No & $24(96 \%)$ & $22(880 \%)$ & $19(76 \%)$ & $25(100 \%)$ & & & \\
\hline \multicolumn{8}{|l|}{ DR } \\
\hline Yes & $3(12 \%)$ & $11(44 \%)$ & $16(64 \%)$ & $0(0 \%)$ & Fisher's exact test & $<0.001^{* *}$ & $\mathrm{G} 2 ; 3>\mathrm{G} 1 ; 4$ \\
\hline No & $22(88 \%)$ & $14(56 \%)$ & $9(36 \%)$ & $25(100 \%)$ & & & \\
\hline \multicolumn{8}{|l|}{ DNeuro } \\
\hline Yes & 16 64\%) & $23(92 \%)$ & 25 (100\%) & $0(0 \%)$ & Fisher's exact test & $<0.001^{* *}$ & $\mathrm{G} 2 ; 3>\mathrm{G} 1 ; 4$ \\
\hline No & $9(36 \%)$ & $2(8 \%)$ & $0(0 \%)$ & 25 (100\%) & & & \\
\hline
\end{tabular}

Categorical variables are expressed as number (\%); continuous variables are expressed as mean \pm standard deviation or median (interquartile range); *significant $(p<0.05)$; **highly significant $(p<0.01)$

$\chi^{2}$ - Chi-square test; ANOVA - analysis of variance; BMI - body mass index; CAD — coronary artery disease; DBP/MBP/SBP — diastolic/mean/systolic blood pressure; DNeuro - diabetic neuropathy; DOD — duration of diabetes; DR - diabetic retinopathy; KWT — Kruskal Wallis test; PAD — peripheral arterial disease; UACR — urinary albumin/creatinine ratio; WC — waist circumference

prehensive scientific database (LncBook) housing more than 270,000 human IncRNAs, PVT1 is the only IncRNA listed as having a validated association with DKD [25]. PVT1 transcript is abundantly expressed in kidney cells and studies have disclosed several plausible mechanisms for its role in DKD pathogenesis. It plays a role in cell cycle progression, cellular transformation, and apoptosis. It possibly promotes mesangial cell expansion by attenuating restraints controlling cell division [11]. It also activates the transforming growth factorbeta (TGFb)/SMAD pathway, a major effector pathway causing epithelial-mesenchymal transition, extracellular matrix accumulation, leading to parenchymal fibrosis and DKD progression [26].

Taken together, there is accumulating evidence of a strong association between PVT1 and DKD occurrence and progression. The present study matches with this evidence and sets forth the possibility that PVT1 might be particularly upregulated in early DKD, compared with the other T2DM patients' groups (Fig. 1). If confirmed, PVT1 might become an ideal novel serum marker of DKD, permitting identification of patients 
Table 3. Laboratory Parameters of the Four Study Groups

\begin{tabular}{|c|c|c|c|c|c|c|c|}
\hline Parameter & $\begin{array}{c}\text { G1 } \\
\text { (normal UACR) }\end{array}$ & $\begin{array}{l}\text { G2 (moderately } \\
\text { increased UACR) }\end{array}$ & $\begin{array}{c}\text { G3 (severely } \\
\text { increased UACR) }\end{array}$ & $\begin{array}{c}\text { G4 } \\
\text { (controls) }\end{array}$ & $\begin{array}{c}\text { Statistical } \\
\text { test }\end{array}$ & $\mathbf{P}$ & $\begin{array}{c}\text { Pairwise } \\
\text { comparisons }\end{array}$ \\
\hline $\begin{array}{l}\text { Hemoglobin } \\
\text { [gm/dL] }\end{array}$ & $13.4 \pm 1.4$ & $12.7 \pm 2.7$ & $11.5 \pm 0.4$ & $15.7 \pm 0.8$ & ANOVA & $<0.001^{* *}$ & $\begin{array}{c}\text { G4 > others } \\
\text { G1 > G3 }\end{array}$ \\
\hline WBCs [1000/uL] & $5(4.2-5.7)$ & $7.8(5.2-9.6)$ & $5.9(5.3-8.1)$ & $5.2(4.7-6.7)$ & KWT & $<0.001 * *$ & $\mathrm{G} 1<\mathrm{G} 2 ; 3$ \\
\hline $\begin{array}{l}\text { Platelets } \\
{[1000 / u L]}\end{array}$ & $236(213-269)$ & $200(225-320)$ & $239(196-304)$ & $214(196-239)$ & KWT & 0.226 & \\
\hline FSG [mg/dL] & $141(120-170)$ & $146(127-195)$ & $167(145-203)$ & $82(81-89)$ & KWT & $<0.001^{* *}$ & G4 < others \\
\hline $\mathrm{HbA} 1 \mathrm{c}[\%]$ & $8.1 \pm 1.5$ & $8.8 \pm 2.2$ & $8.9 \pm 1.8$ & $5 \pm 0.3$ & ANOVA & $<0.001 * *$ & $\mathrm{G} 4<$ others \\
\hline S. TC [mg/dL] & $185.6 \pm 60.4$ & $157.6 \pm 37.1$ & $189.8 \pm 65.6$ & $176.8 \pm 68.1$ & ANOVA & 0.23 & \\
\hline S. TG [mg/dL] & $118(105-188)$ & $126(110-135)$ & $152(104-184)$ & $140(103-180)$ & KWT & 0.804 & \\
\hline S. LDL [mg/dL] & $115(80-135)$ & $102(79-112)$ & $117(75-158)$ & $108(50-153)$ & KWT & 0.333 & \\
\hline S. HDL [mg/dL] & $38(34-43)$ & $40(35-43)$ & $42(33-55)$ & $36(33-43)$ & KWT & 0.379 & \\
\hline S. ALT [U/mL] & $24(20-32)$ & $27(19-33)$ & 24 (19-26) & $21(19-24)$ & KWT & 0.291 & \\
\hline S. AST [U/mL] & $22(19-29)$ & $25(19-33)$ & $25(21-31)$ & $20(18-27)$ & KWT & 0.234 & \\
\hline $\begin{array}{l}\text { S. albumin } \\
\text { [gm/dL] }\end{array}$ & $4.1(3.8-4.3)$ & $3.9(3.4-4.1)$ & $3.5(2.8-3.9)$ & $4.2(4.1-4.3)$ & KWT & $<0.001^{* *}$ & $\begin{array}{c}\mathrm{G} 4>\mathrm{G} 2 ; 3 \\
\mathrm{G} 1>\mathrm{G} 3\end{array}$ \\
\hline S. Urea [mg/dL] & $32(28-37)$ & $40(32-57)$ & 55 (38-79) & $30(29-31)$ & KWT & $<0.001 * *$ & $\mathrm{G} 2 ; 3>\mathrm{G} 1 ; 4$ \\
\hline $\begin{array}{l}\text { S. greatinine } \\
{[\mathrm{mg} / \mathrm{dL}]}\end{array}$ & $0.8(0.7-1)$ & $1.1(0.9-1.3)$ & $1.7(1.5-2)$ & $0.8(0.7-0.8)$ & KWT & $<0.001^{* *}$ & $\begin{aligned} \mathrm{G} 3 & >\text { others } \\
\mathrm{G} 2 & >\mathrm{G} 1 ; 4\end{aligned}$ \\
\hline $\begin{array}{l}\text { eGFR }[\mathrm{mL} / \\
\left.\mathrm{min} / 1.73 \mathrm{~m}^{2}\right]\end{array}$ & $90.3(72.2-100.1)$ & $64.6(50.3-79.7)$ & $36.7(33-43)$ & $\begin{array}{c}108.3(99.8- \\
112.2)\end{array}$ & KWT & $<0.001^{* *}$ & $\begin{array}{c}\mathrm{G} 3<\text { others } \\
\mathrm{G} 2<\mathrm{G} 4\end{array}$ \\
\hline UACR [mg/gm] & $11.4(8.9-19)$ & $110(77-163)$ & $2644(1400-3902)$ & $9(7-11)$ & KWT & $<0.001^{* *}$ & $\begin{array}{l}\mathrm{G} 3>\text { others } \\
\mathrm{G} 2>\mathrm{G} 1 ; 4\end{array}$ \\
\hline Log S. MALAT1 & $-0.212 \pm 0.718$ & $-0.015 \pm 0.452$ & $-0.225 \pm 0.548$ & $-0.173 \pm 0.671$ & ANOVA & 0.603 & \\
\hline Log S. CASC2 & $-0.081 \pm 0.677$ & $-0.351 \pm 0.979$ & $-0.186 \pm 0.781$ & $-0.019 \pm 0.643$ & ANOVA & 0.464 & \\
\hline \multirow[t]{3}{*}{ Log S. PVT1 } & $0.211 \pm 1.15$ & $0.345 \pm 0.427$ & $0.112 \pm 0.397$ & $-0.055 \pm 0.433$ & ANOVA & 0.235 & \\
\hline & \multicolumn{2}{|c|}{ T2DM patients $0.223 \pm 0.747$} & \multicolumn{2}{|c|}{ Controls $-0.019 \pm 0.643$} & T test & $0.033^{*}$ & \\
\hline & \multicolumn{2}{|c|}{ G2 $0.345 \pm 0.427$} & \multicolumn{2}{|c|}{ G1 and G3 $0.163 \pm 086$} & $\mathrm{~T}$ test & $0.024^{*}$ & \\
\hline
\end{tabular}

Categorical variables are expressed as numbers (\%); continuous variables are expressed as mean \pm SD or median (interquartile range); *significant $(p<0.05) ; * *$ highly significant $(p<0.01)$

ALT - alanine transferase; ANOVA — analysis of variance; AST — aspartate transferase; CASC2 — cancer susceptibility candidate 2; eGFR — estimated glomerular filtration rate by CKD-EPI equation; FSG — fasting serum glucose; HDL — high-density lipoproteins; KWT — Kruskal Wallis test; LDL — low-density lipoproteins; MALAT1 — metastasis-associated lung adenocarcinoma transcript 1; PVT1 — plasmacytoma variant translocation 1 gene; S. — serum; TC — total cholesterol; TG - triglycerides; UACR — urinary albumin/creatinine ratio; WBCs - white blood cells

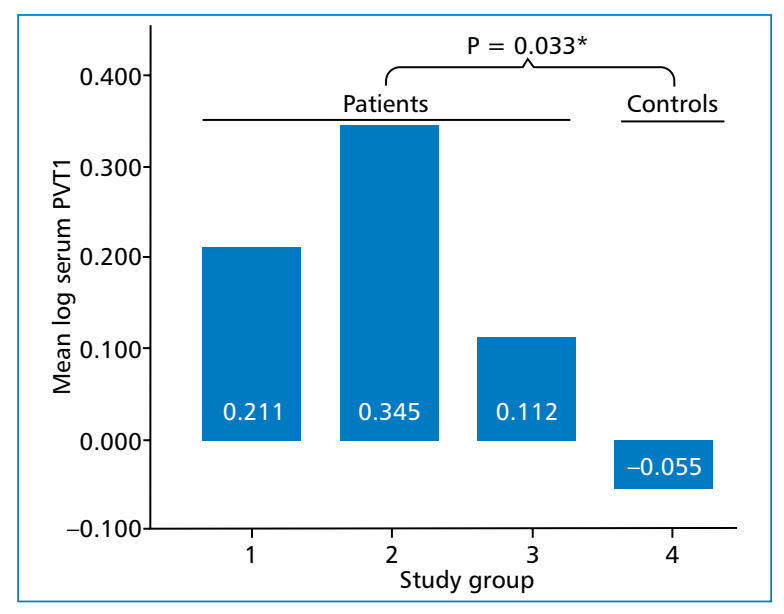

Figure 1. Plasmacytoma Variant Translocation 1 Gene (PVT1) Relative Serum Expression Levels at the highest risk for progression to ESRD at an early disease stage, when potential preventive strategies are most effective.

Dysregulation of IncRNAs might also be involved in the pathogenesis of other diabetic complications. For example, PVT1 and MALAT1 upregulation have been implicated in the pathogenesis of diabetic cataract [27] and diabetic retinopathy [28], respectively. The current study did not reveal any association of MALAT1 or CASC2 with the occurrence of T2DM, DKD, or other major micro- or macrovascular diabetic complications. It is obvious that discrepant findings are not uncommon among IncRNA disease association studies [14]. By a rigorous look at these studies, several explanations can be pursued. First, a great deal of these studies were performed in in-vitro mod- 
Table 4. Association of Abnormal IncRNA Expression with the Occurrence of Diabetes Mellitus (within All Study Subjects; $\mathrm{n}=100)$

\begin{tabular}{lccc}
\hline IncRNA abnormality & Odds ratio & \multicolumn{2}{c}{ 95\% confidence interval } \\
\cline { 3 - 4 } & & Lower boundary & Upper boundary \\
\hline Increased PVT1 & 2.042 & 0.8 & 5.213 \\
Increased MALAT1 & 0.918 & 0.368 & 2.29 \\
Decreased CASC2 & 1.002 & 0.404 & 0.150 \\
\hline
\end{tabular}

CASC2 - cancer susceptibility candidate 2 ; IncRNA — long non-coding RNA; MALAT1 — metastasis-associated lung adenocarcinoma transcript 1; PVT1 — plasmacytoma variant translocation 1 gene

Table 5. Association of Abnormal IncRNA Expression with the Occurrence of Diabetic Complications (within Patients; $\mathrm{n}=75$ )

\begin{tabular}{|c|c|c|c|c|c|}
\hline \multirow[t]{2}{*}{ IncRNA abnormality } & \multirow[t]{2}{*}{ Diabetic complications } & \multirow[t]{2}{*}{ Odds ratio } & \multicolumn{2}{|c|}{$95 \%$ confidence interval } & \multirow[t]{2}{*}{$\mathbf{P}$} \\
\hline & & & Lower boundary & Upper boundary & \\
\hline \multirow[t]{7}{*}{ Increased PVT1 } & Albuminuria & 2.115 & 0.767 & 5.833 & 0.144 \\
\hline & Renal impairment & & & & \\
\hline & $\left(\mathrm{eGFR}<60 \mathrm{~mL} / \mathrm{min} / \mathrm{m}^{2}\right)$ & 0.634 & 0.264 & 1.518 & 0.304 \\
\hline & Diabetic retinopathy & 0.513 & 0.328 & 1.747 & 0.513 \\
\hline & Diabetic neuropathy & 0.711 & 0.311 & 1.624 & 0.417 \\
\hline & CAD & 1.388 & 0.429 & 4.490 & 0.584 \\
\hline & PAD & 0.39 & 0.078 & 1.946 & 0.237 \\
\hline \multirow[t]{7}{*}{ Increased MALAT1 } & Albuminuria & 1.125 & 0.422 & 2.997 & 0.814 \\
\hline & Renal impairment & & & & \\
\hline & $\left(\mathrm{eGFR}<60 \mathrm{~mL} / \mathrm{min} / \mathrm{m}^{2}\right)$ & 1.131 & 0.495 & 2.585 & 0.77 \\
\hline & Diabetic retinopathy & 0.788 & 0.349 & 1.777 & 0.565 \\
\hline & Diabetic neuropathy & 1.192 & 0.537 & 2.649 & 0.666 \\
\hline & CAD & 1.388 & 0.429 & 4.490 & 0.584 \\
\hline & PAD & 3.265 & 0.656 & 16.251 & 0.13 \\
\hline \multirow[t]{7}{*}{ Decreased CASC2 } & Albuminuria & 1.636 & 0.618 & 4.334 & 0.32 \\
\hline & Renal impairment & & & & \\
\hline & $\left(\mathrm{eGFR}<60 \mathrm{~mL} / \mathrm{min} / \mathrm{m}^{2}\right)$ & 0.954 & 0.419 & 2.172 & 0.911 \\
\hline & Diabetic retinopathy & 1.569 & 0.698 & 3.527 & 0.275 \\
\hline & Diabetic Neuropathy & 0.668 & 0.301 & 1.481 & 0.32 \\
\hline & $C A D$ & 0.787 & 0.251 & 2.464 & 0.68 \\
\hline & PAD & 1.097 & 0.252 & 4.765 & 0.902 \\
\hline
\end{tabular}

CAD - coronary artery disease; CASC2 - cancer susceptibility candidate 2; eGFR - estimated glomerular filtration rate; IncRNA — long non-coding RNA; MALAT1 - metastasis-associated lung adenocarcinoma transcript 1; PAD — peripheral arterial disease; PVT1 — plasmacytoma variant translocation 1 gene

els or experimental animals; so their results may not be readily extrapolated to humans. Second, human studies were very divergent regarding the body tissue or fluid studied, the method of IncRNA analysis, the expression of IncRNA level, and even the nomenclature of the IncRNA themselves, making pooled analysis of data difficult [14]. Third, stratification of human studies by the target tissue/fluid results in multiple study groups, each having a relatively low statistical power. More importantly, a significant proportion of studies yielded really discordant results. Indeed, among 638 IncRNAs that were differentially expressed between diabetic patients and controls in different studies, only 6 IncRNAs (<1\%) were consistently dysregulated (significantly higher or lower in cases compared with controls in $\geq 3 / 4$ of eligible studies) [14]. Although this pooled analysis reported MALAT1 as consistently upregulated in diabetic patients, we found no significant differences in serum MALAT1 expression levels among controls and diabetic patients stratified by UACR; and a recent study found MALAT1 to be even significantly reduced in sera of 60 T2DM patients compared with 60 control subjects [29]. Fourth, the impact of diabetic complications, particularly DKD, on IncRNA expression, 
Table 6. Correlations of Serum IncRNAs Expression Levels within Diabetic Patients $(n=75)$

\begin{tabular}{lcccccc}
\hline Parameters & \multicolumn{2}{c}{ S. PVT1 } & \multicolumn{2}{c}{ S. MALAT1 } & \multicolumn{2}{c}{ S. CASC2 } \\
\cline { 2 - 7 } & $\mathbf{r}$ & $\mathbf{p}$ & $\mathbf{r}$ & $\mathbf{p}$ & $\mathbf{p}$ & 0.431 \\
\hline Age & -0.108 & 0.364 & 0.187 & 0.110 & 0.094 & 0.162 \\
BMI & -0.087 & 0.466 & 0.096 & 0.415 & 0.165 & 0.323 \\
DOD & -0.006 & 0.961 & 0.025 & 0.83 & -0.117 & 0.899 \\
SBP & -0.089 & 0.454 & 0.073 & 0.534 & -0.015 & 0.771 \\
DBP & -0.022 & 0.853 & 0.14 & 0.235 & -0.035 & 0.71 \\
MBP & -0.042 & 0.722 & 0.117 & 0.32 & -0.044 & 0.636 \\
Hemoglobin & -0.05 & 0.676 & -0.057 & 0.628 & 0.056 & 0.564 \\
WBC & 0.116 & 0.327 & -0.109 & 0.356 & -0.069 & 0.371 \\
Platelets & 0.09 & 0.449 & -0.088 & 0.457 & -0.106 & 0.804 \\
FSG & -0.093 & 0.436 & -0.125 & 0.287 & -0.03 & 0.838 \\
HbA1c & -0.037 & 0.753 & -0.077 & 0.517 & -0.024 & 0.154 \\
S. TC & -0.204 & 0.084 & -0.188 & 0.108 & -0.169 & 0.713 \\
S. TG & -0.114 & 0.338 & -0.121 & 0.304 & -0.044 & 0.282 \\
S. LDL & -0.185 & 0.118 & -0.202 & 0.084 & -0.128 & 0.864 \\
S. HDL & -0.044 & 0.711 & 0.157 & 0.18 & -0.02 & 0.85 \\
S. ALT & 0.124 & 0.297 & -0.079 & 0.505 & -0.022 & 0.206 \\
S. AST & -0.069 & 0.564 & -0.05 & 0.672 & -0.15 & 0.434 \\
S. Albumin & -0.109 & 0.358 & -0.019 & 0.875 & 0.093 & 0.277 \\
S. Urea & 0.008 & 0.946 & 0.035 & 0.77 & -0.129 & 0.785 \\
S. Creatinine & -0.141 & 0.236 & 0.009 & 0.94 & -0.032 & 0.701 \\
eGFR & 0.137 & 0.246 & -0.048 & 0.684 & 0.046 & 0.465 \\
UACR & -0.096 & 0.419 & 0.009 & 0.941 & -0.087 & \\
\hline
\end{tabular}

*significant $(p<0.05)$; **highly significant $(p<0.01)$

ALT — alanine transferase; AST — aspartate transferase; BMI — body mass index; CASC2 — cancer susceptibility candidate 2; DBP/MBP/SBP — diastolic/ mean/systolic/blood pressure; DOD — duration of diabetes; eGFR — estimated glomerular filtration rate; FSG — fasting serum glucose; HbA1c — glycated hemoglobin; HDL 5- high-density lipoproteins; LDL - low-density lipoproteins; MALAT1 - metastasis-associated lung adenocarcinoma transcript 1; PVT1 - plasmacytoma variant translocation 1 gene; $r$ - Spearman correlation coefficient; S. - serum; WBCs - white blood cells; TC - total cholesterol; TG — triglycerides; UACR — urinary albumin/creatinine ratio

Table 7. Multiple Linear Regression Analysis for Predictors of Increased UACR Among Diabetic Patients (Groups 1, 2, 3) $(n=75)$

\begin{tabular}{|c|c|c|c|c|c|}
\hline \multirow[t]{2}{*}{ Variable } & \multicolumn{2}{|c|}{ Unstandardized coefficients } & \multirow{2}{*}{$\begin{array}{c}\text { Standardized coefficients } \\
\text { Beta }\end{array}$} & \multirow[t]{2}{*}{$\mathbf{t}$} & \multirow[t]{2}{*}{ Sig. } \\
\hline & B & Std. error & & & \\
\hline (Constant) & -3746.016 & 2070.113 & & -1.81 & 0.075 \\
\hline DOD & 51.562 & 27.004 & 0.201 & 1.909 & 0.06 \\
\hline MBP & 5.132 & 18.321 & 0.031 & 0.28 & 0.78 \\
\hline BMI & 35.971 & 36.153 & 0.11 & 0.995 & 0.323 \\
\hline $\mathrm{HbA1c}$ & 74.982 & 110.206 & 0.074 & 0.68 & 0.499 \\
\hline Serum creatinine & 1582.015 & 494.014 & 0.385 & 3.202 & $0.002 * *$ \\
\hline
\end{tabular}

Model $R^{2}=0.305 ;$ model significance: $p<0.001$

$\mathrm{BMI}$ - body mass index; $\mathrm{HbA}_{1 \mathrm{c}}$ - glycated hemoglobin; $\mathrm{DOD}$ - duration of diabetes; MBP — mean blood pressure; UACR — urinary albumin/creatinine ratio

was not adequately clarified by many studies. CKD in the context of T2DM tended to be automatically equated with DKD; whereas recent findings denote a trend for an increased prevalence of non-DKD (NDKD) in T2DM patients, particularly among the elderly and the normoalbuminuric patients. NDKD may have totally different pathogenesis and IncRNA profile [30]. Fifth, patients with true DKD still constitute a heterogeneous group, with different trajectories of kidney function decline and proteinuria/albuminuria. Recently, five 
different classes of DKD have been described based on cluster analysis of 6 variables [31]. These divergent phenotypes may have divergent pathogenesis and IncRNA trajectories [5]. Sixth, studies are largely based on glomerulocentric view of DKD pathogenesis, with glomerular hyperfiltration and protein leak viewed as the initial inciting event. Indeed, recent insights into DKD pathogenesis favor a more tubulocentric view; so the markers of tubular function and damage should ideally be reported hand in hand with the classical parameters of UACR and eGFR [32]. Seventh, IncRNA are not independently functioning molecules, but are pivotal members of several, complex, multi-level interactive control circuits; each having a host of upstream and downstream tightly regulated signaling pathways. For example, PVT1 signals through miR-23b-3p/WT1, miR-23b-3p/EGR1, miR-1207-5P/TGFb/SMAD or FOXA1 (hepatocyte nuclear factor 3-alpha) pathways [26]. MALAT1 signals through bone morphogenetic proteins (BMPs), Wnt/beta-catenin, or mitogen-activated protein kinases/extracellular signal-regulated kinases (MAPK/ ERK) pathways. CASC2 functions through miR-9-5p/ PPAR $\gamma$ or miR-133b/FOXP1 axes. Inter-individual variations in the signaling pathway activities are partially genetically-determined and may significantly affect the response to a given circulating IncRNA level [33]. IncRNA themselves might have SNPs leading to different biological activities and phenotypic expression [11, 22]. Even identical IncRNA genetic isoforms were found to have different disease associations in cohorts of different ethnicities. This was attributed to substantial differences in linkage disequilibrium and genetic background [22]. Last, and probably of major importance, UACR (and to a lesser extent eGFR) do vary over time, and so a single, or few, cross-sectional determinations of these parameters would not suffice to express their longitudinal trajectories or time-averaged values, which may be more relevant to the circulating IncRNA levels [6, 34].

Human IncRNA studies in Middle Eastern populations are sparse. We studied serum samples and confirmed their feasibility for IncRNA research. Patients were classified into well-defined study groups to allow tracing the influence of selected IncRNAs across the typical DKD progression stages. We acknowledge some limitations. The study's cross-sectional nature and the relatively limited number of participants might have undermined our ability to clinch statistical evidence for potential IncRNAs associations with diabetes or its complications. Contrary to our plan, the control subjects available for inclusion were significantly younger than the patients. Among the IncRNAs studied, however, only MALAT1 is senescence-associated (decreases with aging) [35]. Assuming a lower serum MALAT1 in control subjects would approximate them towards two of the three patients' groups, further drawing MALAT1 association with DKD towards the null hypothesis, and not affecting the validity of the results. Finally, we did not check for key downstream mediators involved in DKD pathogenesis, e.g., TGFb.

\section{Conclusions}

The IncRNA PVT1 is a reliable early serum biomarker of DKD, permitting early identification of those at the highest risk for progression to ESRD. It may also provide a plausible therapeutic approach, when PVT1 antisense oligonucleotides, for instance, can be delivered to the kidney by carrier exosomes or gold nanoparticles. We could not replicate previous findings that MALAT1 upregulation or CASC2 downregulation are involved in DKD occurrence and progression. A long road remains before the pathophysiologic roles of various IncRNAs in diabetes and its complications become adequately clarified. Further large-scale, longitudinal studies should explore various IncRNAs as well as their upstream and downstream signaling pathways; and should accommodate the different DKD phenotypes and consider both the gomerulocentric and the tubulocentric views of DKD pathogenesis.

\section{Conflict of interest}

None declared.

\section{REFERENCES}

1. American Diabetes Association. 2. Classification and diagnosis of diabetes: standards of medical care in diabetes-2021. Diabetes Care. 2021; 44(Suppl 1): S15-S33, doi: 10.2337/dc21-S002, indexed in Pubmed: 33298413.

2. IDF Diabetes Atlas, 9th Ed. Dunia 2019.

3. Shelbaya S, Halawa M, Nasr M. The management of care of egyptian patients with diabetes: a report from the international diabetes management practices study wave 7. Med. J. Cairo Univ. 2020; 88(3): 1413-1421, doi: 10.21608/mjcu.2020.110954.

4. Macisaac RJ, Ekinci El, Jerums G. Markers of and risk factors for the development and progression of diabetic kidney disease. Am J Kidney Dis. 2014; 63(2 Suppl 2): S39-S62, doi: 10.1053/j. ajkd.2013.10.048, indexed in Pubmed: 24461729.

5. Yamanouchi M, Furuichi K, Hoshino J, et al. Research Group of Diabetic Nephropathy, the Ministry of Health, Labour and Welfare, and the Japan Agency for Medical Research and Development. Two-year longitudinal trajectory patterns of albuminuria and subsequent rates of end-stage kidney disease and all-cause death: a nationwide cohort study of biopsy-proven diabetic kidney disease. BMJ Open Diabetes Res Care. 2021; 9(1), doi: 10.1136/ bmjdrc-2021-002241, indexed in Pubmed: 34385147.

6. Leong A, Ekinci El, Nguyen C, et al. Long-term intra-individual variability of albuminuria in type 2 diabetes mellitus: implications for categorization of albumin excretion rate. BMC Nephrol. 2017; 18(1): 355, doi: 10.1186/s12882-017-0767-3, indexed in Pubmed: 29207965.

7. Gu HF. Genetic and epigenetic studies in diabetic kidney disease. Front Genet. 2019; 10: 507, doi: 10.3389/fgene.2019.00507, indexed in Pubmed: 31231424. 
8. Lv J, Wu Yu, Mai Y, et al. Noncoding rnas in diabetic nephropathy: pathogenesis, biomarkers, and therapy. J Diabetes Res. 2020; 2020: 3960857, doi: 10.1155/2020/3960857, indexed in Pubmed: 32656264.

9. Li $Y, X u K, X u K$, et al. Roles of identified long noncoding RNA in diabetic nephropathy. J Diabetes Res. 2019; 2019: 5383010, doi: 10.1155/2019/5383010, indexed in Pubmed: 30891461.

10. Gu YY, Lu FH, Huang XR, et al. Non-coding RNAs as biomarkers and therapeutic targets for diabetic kidney disease. Front Pharmacol. 2021; 11: 583528, doi: 10.3389/fphar.2020.583528, indexed in Pubmed: 33574750

11. Hanson RL, Craig DW, Millis MP, et al. Identification of PVT1 as a candidate gene for end-stage renal disease in type 2 diabetes using a pooling-based genome-wide single nucleotide polymorphism association study. Diabetes. 2007; 56(4): 975-983, doi: 10.2337/db06-1072, indexed in Pubmed: 17395743.

12. Li X, Zeng Li, Cao C, et al. Long noncoding RNA MALAT1 regulates renal tubular epithelial pyroptosis by modulated miR-23c targeting of ELAVL1 in diabetic nephropathy. Exp Cell Res. 2017; 350(2): 327-335, doi: 10.1016/j.yexcr.2016.12.006, indexed in Pubmed: 27964927.

13. Zhang $X L$, Zhu HQ, Zhang $Y$, et al. LncRNA CASC2 regulates high glucose-induced proliferation, extracellular matrix accumulation and oxidative stress of human mesangial cells via miR-133b/FOXP1 axis. Eur Rev Med Pharmacol Sci. 2020; 24(2): 802-812, doi: 10.26355/eurrev_202001_20063, indexed in Pubmed: 32016985 .

14. Dieter C, Lemos NE, Corrêa NR, et al. The Impact of IncRNAs in Diabetes Mellitus: A Systematic Review and Analyses. Front Endocrinol (Lausanne). 2021; 12: 602597, doi: 10.3389/fendo.2021.602597, indexed in Pubmed: 33815273.

15. Quinn JJ, Chang HY. Unique features of long non-coding RNA biogenesis and function. Nat Rev Genet. 2016; 17(1): 47-62, doi: 10.1038/nrg.2015.10, indexed in Pubmed: 26666209.

16. Tuttle KR, Bakris GL, Bilous RW, et al. Diabetic kidney disease: a report from an ADA Consensus Conference. Diabetes Care. 2014; 37(10): 2864-2883, doi: 10.2337/dc14-1296, indexed in Pubmed: 25249672.

17. Lopez J, Burtis CA, Bruns DE. Tietz fundamentals of clinical chemistry and molecular diagnostics. Springer 2015.

18. van den Brand JA, van Boekel GAJ, Willems HL, et al. Introduction of the CKD-EPI equation to estimate glomerular filtration rate in a Caucasian population. Nephrol Dial Transplant. 2011; 26(10): 3176-3181, doi: 10.1093/ndt/gfr003, indexed in Pubmed: 21325352.

19. Desjardins P, Conklin D. NanoDrop microvolume quantitation of nucleic acids. J Vis Exp. 2010(45): 2565, doi: 10.3791/2565, indexed in Pubmed: 21189466.

20. Livak KJ, Schmittgen TD. Analysis of relative gene expression data using real-time quantitative PCR and the 2(-Delta Delta $C(T)$ ) Method. Methods. 2001; 25(4): 402-408, doi: 10.1006/ meth.2001.1262, indexed in Pubmed: 11846609.

21. Changyong F, Hongyue W, Naiji L, et al. Log-transformation and its implications for data analysis. Shanghai Arch Psychiatry. 2014; 26(2): 105-109, doi: 10.3969/j.issn.1002-0829.2014.02.009, indexed in Pubmed: 25092958.

22. Millis MP, Bowen D, Kingsley C, et al. Variants in the plasmacytoma variant translocation gene (PVT1) are associated with end-stage renal disease attributed to type 1 diabetes. Diabetes.
2007; 56(12): 3027-3032, doi: 10.2337/db07-0675, indexed in Pubmed: 17881614.

23. Yu D, Yang $X$, Zhu Y, et al. Knockdown of plasmacytoma variant translocation 1 (PVT1) inhibits high glucose-induced proliferation and renal fibrosis in HRMCs by regulating miR-23b-3p/early growth response factor 1 (EGR1). Endocr J. 2021; 68(5): 519-529, doi: 10.1507/endocrj. EJ20-0642, indexed in Pubmed: 33408314.

24. Zhong W, Zeng J, Xue J, et al. Knockdown of IncRNA PVT1 alleviates high glucose-induced proliferation and fibrosis in human mesangial cells by miR-23b-3p/WT1 axis. Diabetol Metab Syndr. 2020; 12: 33, doi: 10.1186/s13098-020-00539-x, indexed in Pubmed: 32322310.

25. Ma L, Cao J, Liu L, et al. LncBook: a curated knowledgebase of human long non-coding RNAs. Nucleic Acids Res. 2019; 47(D1): D128-D134, doi: 10.1093/nar/gky960, indexed in Pubmed: 30329098.

26. Alvarez ML, Khosroheidari M, Eddy E, et al. Role of microRNA 1207$5 \mathrm{P}$ and its host gene, the long non-coding RNA Pvt1, as mediators of extracellular matrix accumulation in the kidney: implications for diabetic nephropathy. PLoS One. 2013; 8(10): e77468, doi: 10.1371/ journal.pone.0077468, indexed in Pubmed: 24204837.

27. Yang J, Zhao S, Tian F. SP1-mediated IncRNA PVT1 modulates the proliferation and apoptosis of lens epithelial cells in diabetic cataract via miR-214-3p/MMP2 axis. J Cell Mol Med. 2020; 24(1): 554-561, doi: 10.1111/jcmm.14762, indexed in Pubmed: 31755246.

28. Radhakrishnan R, Kowluru RA. Long noncoding RNA and regulation of the antioxidant defense system in diabetic retinopathy. Diabetes. 2021; 70(1): 227-239, doi: 10.2337/db20-0375, indexed in Pubmed: 33051272.

29. Tello-Flores VA, Valladares-Salgado A, Ramírez-Vargas MA, et al. Altered levels of MALAT1 and H19 derived from serum or serum exosomes associated with type-2 diabetes. Noncoding RNA Res. 2020; 5(2): 71-76, doi: 10.1016/j.ncrna.2020.03.001, indexed in Pubmed: 32346662.

30. Anders HJ, Huber TB, Isermann B, et al. CKD in diabetes: diabetic kidney disease versus nondiabetic kidney disease. Nat Rev Nephrol. 2018; 14(6): 361-377, doi: 10.1038/s41581-018-0001-y, indexed in Pubmed: 29654297.

31. Ahlqvist $E$, Storm $P$, Käräjämäki $A$, et al. Novel subgroups of adultonset diabetes and their association with outcomes: a data-driven cluster analysis of six variables. Lancet Diabetes Endocrinol. 2018; 6(5): 361-369, doi: 10.1016/S2213-8587(18)30051-2, indexed in Pubmed: 29503172.

32. Zeni L, Norden AGW, Cancarini G, et al. A more tubulocentric view of diabetic kidney disease. J Nephrol. 2017; 30(6): 701-717, doi: 10.1007/s40620-017-0423-9, indexed in Pubmed: 28840540.

33. Sharma G, Sharma AR, Seo EM, et al. Genetic polymorphism in extracellular regulators of Wnt signaling pathway. Biomed Res Int. 2015; 2015: 847529, doi: 10.1155/2015/847529, indexed in Pubmed: 25945348.

34. Waikar SS, Rebholz CM, Zheng Z, et al. Chronic Kidney Disease Biomarkers Consortium Investigators. Biological variability of estimated GFR and albuminuria in CKD. Am J Kidney Dis. 2018; 72(4): 538-546, doi: 10.1053/j.ajkd.2018.04.023, indexed in Pubmed: 30031564.

35. Abdelmohsen K, Panda A, Kang MJ, et al. Senescence-associated IncRNAs: senescence-associated long noncoding RNAs. Aging Cell. 2013; 12(5): 890-900, doi: 10.1111/acel.12115, indexed in Pubmed: 23758631. 